\title{
REVIEW
}

\section{POTENCIALIDADES DE LAS MACROALGAS MARINAS ARGENTINAS}

\author{
Julieta R. CAmurati ${ }^{1}$, Julieta Hocsman ${ }^{1}$ y VANesa N. SAlomone ${ }^{1,2,3}$ \\ ${ }^{1}$ Instituto de Investigación e Ingeniería Ambiental (IIIA), \\ Universidad Nacional de San Martín (UNSAM-CONICET), \\ Campus Miguelete, Av. 25 de Mayo y Francia, 1650 - San Martín, Argentina \\ ${ }^{2}$ Consejo Nacional de Investigaciones Científicas y Técnicas (CONICET), Argentina \\ ${ }^{3}$ correo electrónico: vsalomone@unsam.edu.ar
}

\begin{abstract}
RESUMEN. En la extensa costa argentina se encuentran numerosas especies de macroalgas de importancia a nivel mundial. En el presente trabajo se debaten los estudios más relevantes publicados hasta la fecha sobre los usos de las algas marinas y se mencionan las investigaciones argentinas con el objetivo de evaluar el estado de conocimiento del recurso. La revisión muestra que en nuestro país se publicaron numerosos trabajos que evidencian el valor de las algas en la alimentación humana y animal, en la producción de compuestos químicos beneficiosos para la salud y en aplicaciones ambientales. Sin embargo, ciertas áreas tales como el desarrollo de biocombustibles y el uso en el campo de la agricultura revelan la necesidad de profundizar en la investigación. Se concluye que las algas marinas argentinas, además de tener numerosas aplicaciones, son un recurso con gran potencial de explotación comercial para beneficio de las poblaciones costeras.
\end{abstract}

Palabras clave: Algas marinas, alimento saludable, biosorción, nutraceútico, biocombustible, fertilizante.

\section{POTENTIALITIES OF ARGENTINE MARINE MACROALGAE}

\begin{abstract}
In the extensive Argentine coast numerous species of macroalgae of importance at global level are found. In this work the most relevant studies published to date on the uses of seaweed are discussed and Argentine researches are mentioned in order to assess the state of knowledge of the resource. The review shows that in our country numerous works that prove the value of algae in human and animal feeding, in the production of chemical compounds beneficial to health and in environmental applications were published. However, certain areas such as the development of biofuels and the use in the agricultural field reveal the need to deepen research. It is concluded that Argentine seaweeds, besides having numerous applications, are a resource with a great potential for commercial exploitation for the benefit of coastal populations.
\end{abstract}

Key words: Seaweeds, healthy food, biosorption, nutraceutical, biofuel, fertilizer.

\section{INTRODUCCIÓN}

Las macroalgas marinas se diferencian en tres grupos: las algas pardas (Phaeophyta), las algas rojas (Rhodophyta) y las algas verdes (Chloro- phyta). La Argentina tiene una costa extensa donde se encuentran gran cantidad de especies de macroalgas. Boraso de Zaixso et al. (2015) describen de manera detallada las algas marinas de importancia económica de la región Patagónica. Allí se destacan algunas especies de gran valor comercial a nivel mundial: Macrocystis pyrifera, 
Lessonia flavicans Bory, Gracilaria gracilis, Pyropia columbina (ex Porphyra) y Undaria pinnatifida. Esta última especie es nativa de Asia y ha invadido exitosamente muchas costas del mundo, incluso las costas de América del Sur, causando daños en los ecosistemas receptores (James 2017). En la Argentina, U. pinnatifida se reportó por primera vez en 1992 en Puerto Madryn (Casas y Piriz 1996) y, a pesar de los esfuerzos realizados para evitar su dispersión, la invasión progresó rápidamente (Martin y Cuevas 2006; Dellatorre et al. 2014). Más tarde fue registrada en las costas de la Provincia de Buenos Aires por Meretta et al. (2012). Experimentos de remoción de individuos de Undaria en el Golfo Nuevo (Patagonia) resultaron en un aumento significativo de la riqueza y diversidad de las algas marinas nativas, evidenciando el impacto negativo de la presencia de esta especie en la Argentina (Casas et al. 2004). En algunos países donde Undaria fue introducida, se ha propuesto su cosecha con fines comerciales como alternativa de control (Boraso de Zaixso et al. 2015). En ese sentido, las algas presentan numerosos usos y aplicaciones en diferentes campos.

La Argentina tiene un gran potencial para la explotación de macroalgas: posee una extensa costa marítima y un recurso abundante con valor comercial. Sin embargo, es un recurso relativamente poco estudiado en comparación con lo que ocurre en otros países de América del Sur, como Chile. Según la Organización de las Naciones Unidas para la Alimentación y la Agricultura (FAO 2018), Chile aparece como uno de los principales productores y exportadores de algas marinas cultivadas de América del Sur, con 15.000 t declaradas en 2016. Además, el comercio de plantas acuáticas, donde predominan las macroalgas, aumentó de 60 millones de USD en 1976 a más de 1.000 millones de USD en 2016 (FAO 2018), lo que demuestra que es un mercado en expansión. Por otra parte, la obtención de algas como materia prima, ya sea de cultivos o extracción directa, se realiza generalmente de manera artesanal con bajo impacto sobre el ecosistema marino. En ese sentido, muchos trabajos señalan que el cultivo de macroalgas tiene un impacto ambiental relativamente bajo comparado con otras formas de acuicultura, debido a que no es necesario añadir nutrientes $u$ otros suplementos (Walls et al. 2017). Por lo tanto, la producción de macroalgas podría desarrollarse de manera ambientalmente sostenible.

El presente trabajo revisa los artículos sobre algas marinas argentinas publicados hasta el momento, con el objetivo de evaluar cuál es el estado del conocimiento sobre este recurso en relación a los usos actuales y las aplicaciones potenciales.

METODOLOGÍA DE LA REVISIÓN

Para llevar adelante el objetivo del trabajo, se realizó una revisión sistemática de la literatura que combina diferentes palabras clave. La metodología consistió en una búsqueda exhaustiva de información, especialmente de la Argentina, en la base de datos bibliográfica SCOPUS, SCIELO y Google Académico, utilizando palabras clave como: algas marinas, macroalgas, combinado con composición química, algas marinas comestible, composición de alimentos, análisis de alimentos, inocuidad de los alimentos, metales pesados, biorremediación, biomonitor, bioindicador, antioxidante, anticancerígeno, biocombustible y biofertilizante.

\section{RESULTADOS}

\section{Aplicaciones alimenticias}

En sus inicios, las algas marinas se cosechaban para la elaboración de espesantes como el alginato, el agar y la carragenina, así como para su con- 
sumo directo. Las macroalgas son consideradas un alimento natural y saludable debido a sus múltiples beneficios. Son ricas en proteínas, vitaminas (sobre todo A, C y B-12), lípidos, fibras y minerales, especialmente los denominados micronutrientes, como el hierro, calcio, yodo, potasio y selenio (Cofrades et al. 2010; Skirzypczyk et al. 2019). También son fuente de ácidos grasos naturales como los omega-3 (Schmid et al. 2018). Son consumidas de manera directa o como suplemento alimenticio, tanto en alimentación humana como animal (Taboada et al. 2013; Rebours et al. 2014; Cofrades et al. 2017). El consumo a nivel mundial se ha incrementado en los últimos años, siendo los países asiáticos, en especial China, Japón y Corea, los principales consumidores (FAO 2018). Algunas de las razones podrían estar relacionadas con ciertos cambios en los hábitos alimenticios, como el aumento de la popularidad de las dietas veganas y vegetarianas y la gastronomía gourmet (Baroni et al. 2019).

La producción mundial de algas marinas aumentó su volumen de 13,5 millones de toneladas en 1995 a algo más de 30 millones de toneladas en 2016 (FAO 2018). Entre las especies más cultivadas se destacan $U$. pinnatifida, Pyropia spp. Porphyra spp. y Gracilaria spp., que se destinan casi exclusivamente al consumo humano directo. Algunos de los formatos de comercialización más usados corresponden al alga cruda deshidratada (rollos, picada, trozada, molida, harina), cocidas o ahumadas, entre otros (Muñoz 2015). En particular en Asia oriental, se utilizan mucho en sopas, y algunas especies (Pyropia y Porphyra) se usan para envolver el sushi. Además, existen en el mercado numerosos alimentos y bebidas con sabor a alga (incluso helados), y se está estudiando el uso de las algas marinas como sustituto de la sal. El principal mercado de esos productos es Asia y el Pacífico, pero también crece el interés en Europa y América del Norte (FAO 2018).

La composición química de las algas marinas es muy variable y depende del grupo, la especie, el momento de la recolección, el hábitat y otras condiciones externas como la temperatura del agua, la intensidad de luz y la concentración de nutrientes en el agua de mar (Balboa et al. 2015; Rodrigues et al. 2015; Astorga-España et al. 2016). Un aspecto fundamental, desde el punto de vista de la salud, es que estos organismos son capaces de absorber y acumular metales tóxicos y metaloides del medio donde se desarrollan (Rubio et al. 2017; Bonanno y Orlando-Bonaca 2018). Muchos trabajos han abordado esta problemática en especial cuando se trata de especies comestibles (Marzocchi et al. 2016; Taylor y Jackson 2016; Miedico et al. 2017; Circuncisáo et al. 2018; Ma et al. 2018; Paz et al. 2018, 2019). Ciertas investigaciones sugieren que la ingesta de metales tóxicos a través del consumo de algas no es despreciable (Taylor et al. 2017; Amin et al. 2018). Es por ello que los estudios de composición elemental de estos organismos, tanto en términos de su valor nutritivo como de su toxicidad, se vuelven indispensables. Sin embargo, hay que tener en cuenta que el contenido de un elemento en un determinado alimento no necesariamente indica la cantidad absorbible de ese analito en el tracto gastrointestinal. Para estimar ese valor deben realizarse experimentos de bioaccesibilidad que simulan la digestión (Alves et al. 2018). Asimismo, hay que mencionar que ciertos métodos de cocción pueden alterar la biodisponibilidad tanto de nutrientes como de elementos tóxicos (García-Sartal et al. 2013).

Respecto a las publicaciones en la Argentina, existen numerosos antecedentes sobre algas para uso alimenticio. Entre los primeros trabajos encontramos el realizado por Muse et al. (1989), quienes determinaron el contenido de arsénico orgánico e inorgánico en algunas especies de algas marinas de la costa de Chubut (M. pyrifera, L. fuscescens, Adenocystis ultricularis, Leathesia difformis, Gigartina skottsbergii, Colpomenia sp.) utilizadas en la elaboración de productos alimenticios para consumo humano y animal. El tra- 
bajo evidencia la gran capacidad de acumulación de arsénico de las algas; sin embargo, los autores aseguran que el contenido de arsénico inorgánico encontrado no representa una amenaza. Como profundizaremos más adelante, la presencia de este elemento potencialmente tóxico en las algas marinas comestibles ha sido ampliamente estudiada debido a las distintas formas en las que puede estar presente y que difieren en su toxicidad (Perez et al. 2010). Pérez et al. (2007) evaluaron el contenido químico en las algas $P$. columbina y Ulva sp. del Golfo San Jorge utilizadas para alimentación. En este caso, los resultados también sugirieron que la ingesta de esas algas no representaba un riesgo para la salud en relación con su contenido de $\mathrm{Cd}$ y $\mathrm{Pb}$. Como se mencionó anteriormente, $U$. pinnatifida es un alga ampliamente estudiada por su extendido uso para consumo humano directo. Gil et al. (2015) evaluaron la composición nutricional y las concentraciones de oligoelementos e hidrocarburos en láminas y esporofitos (estructura reproductiva) de $U$. pinnatifida de Golfo Nuevo (GN) y Golfo San José (GSJ) (Chubut). El trabajo concluye que estas algas marinas son fuente importante de proteínas totales, fibra insoluble, lípidos, carbohidratos (distintos de los polisacáridos estructurales), vitaminas, macrominerales y oligoelementos esenciales; sugiriendo que $U$. pinnatifida de GN y GSJ sería adecuada para el consumo humano y animal como un suplemento dietético natural. También hay reportes sobre la composición química de $U$. pinnatifida y $M$. pyrifera del Golfo San Jorge, en los cuales se reafirman a las algas como fuente de micronutrientes esenciales, pero también evidencian su capacidad para incorporar y acumular elementos tóxicos o potencialmente tóxicos como el arsénico (Salomone et al. 2017; Salomone y Riera 2019). En ese sentido, Camurati y Salomone (2019) publicaron un trabajo en el cual repasan distintos aspectos del arsénico en las algas marinas comestibles incluyendo su incorporación, transformación y efectos sobre la salud humana. Por otra parte, las algas son muy importantes para los ecosistemas marinos y son refugio y alimento de muchos otros organismos. En relación con esto, un estudio de la composición de la biomasa microbiana presente en un alga comestible (Monostroma undulatum) de Puerto Deseado en la Provincia de Santa Cruz, indicó que las cepas encontradas no eran patógenas, por lo que el uso alimenticio de esta especie era aceptable (Gallardo et al. 2004).

Las algas marinas también son utilizadas mundialmente como alimento en el cultivo de crustáceos, moluscos y peces, normalmente en forma de polvo seco, pellets o extractos. En este caso, suelen utilizarse los productos de baja calidad y/o productos residuales resultantes del procesamiento de algas (FAO 2018). Además, las macroalgas tienen una larga historia de uso como alimento para animales de ganado, debido a que son una fuente importante de proteínas con altos niveles de aminoácidos esenciales, como la lisina, que es el aminoácido limitante en los alimentos para animales de corral (Astorga-España et al. 2016; Makkar et al. 2016).

En la Argentina, varios trabajos investigaron el uso de algas para alimentación animal. Díaz et al. (2017) usaron extractos de U. pinnatifida como aditivo alimenticio del camarón (Artemesia longinaris) y registraron una mejora en el crecimiento evidenciado en un mayor peso. Otro estudio evaluó la alimentación del pacú (Piaractus mesopotamicus) con un alga marina roja ( $P$. columbina) del Golfo San Jorge y observaron efectos beneficiosos sobre el metabolismo de los lípidos en los peces con respecto a la dieta control. Los autores concluyen que $P$. columbina podría ser utilizada como un ingrediente antioxidante natural en la alimentación contribuyendo a un mejor estado nutricional de los peces cultivados (Cian et al. 2019).

\section{Aplicaciones ambientales}

Relacionado con la capacidad de estos organismos de sorber elementos no esenciales y otros 
contaminantes del medio circundante, se destacan dos aplicaciones ambientales: el uso de las algas marinas como biomonitores y en remediación.

Los indicadores biológicos proporcionan información sobre los efectos a largo plazo de la contaminación por metales y sobre el potencial impacto a niveles más altos como resultado de las interacciones tróficas. Los organismos que acumulan metales en los tejidos pueden ser particularmente útiles (Farías et al. 2018). El uso de organismos aislados para evaluar la presencia de contaminantes se conoce como biomonitoreo, y dicho organismo se clasifica como "bioindicador" (Zhou et al. 2008). Según Zhou et al. (2008) un bioindicador debe ser: i) capaz de acumular altos niveles de contaminantes; ii) sésil o estar restringido a un lugar determinado para reflejar la contaminación local; iii) relevante en la cadena alimentaria y iv) abundante, y v) fácil de identificar y colectar. Las algas marinas se han utilizado ampliamente para monitorear y caracterizar el estado de la contaminación en ambientes marinos debido a que desempeñan un papel importante en la dinámica de los nutrientes y reflejan los cambios en la calidad del agua de manera eficiente (Karthick et al. 2012). Numerosos estudios han demostrado la relación entre el contenido de metales en sedimentos, agua de mar y algas (Paz et al. 2019).

En la Argentina hay trabajos que evalúan el potencial de las macroalgas como biomonitores. Farías et al. $(2002,2007)$ estudiaron macroalgas antárticas y sus resultados apoyan la hipótesis sobre la capacidad selectiva de las algas para acumular contaminantes inorgánicos del agua de mar, lo que las convierte en buenos monitores ambientales. También se estudió el uso de $U$. lactuca en la evaluación de la contaminación marina en relación con su capacidad para acumular $\mathrm{Cd}$ y $\mathrm{Pb}$ (Muse et al. 2006). La capacidad de las macroalgas de incorporar elementos también ha sido útil en los sistemas de producción acuícola. El rápido desarrollo de la acuicultura alimentaria (por ejemplo, de peces y camarones) en las zonas costeras de todo el mundo ha suscitado una preocupación cada vez mayor por sus efectos ambientales (Diana et al. 2013). Los efluentes de la acuicultura son ricos en nutrientes (nitrógeno, fósforo) y tienen alta carga de materia orgánica. Una alternativa a las prácticas tradicionales radica en desarrollar simultáneamente la piscicultura y la producción de algas marinas. Esto es ambientalmente positivo y puede tener beneficios económicos (Afonso et al. 2018). En ese sentido, investigadores argentinos comprobaron la alta capacidad de $U$. rigida y $U$. pinnatifida para eliminar el nitrógeno y otros nutrientes derivados de aguas residuales de medios de cultivo (Torres et al. 2004; Gil et al. 2005).

La remoción de contaminantes de aguas superficiales, residuales y efluentes requiere de métodos sencillos y económicos, especialmente para aplicaciones a gran escala. Entre las numerosas técnicas utilizadas para la remoción de metales y metaloides de solución acuosa, la adsorción resulta muy efectiva y de bajo costo debido a que pueden usarse materiales económicos, incluso de desecho. Las algas representan un biomaterial muy abundante que ha sido empleado exitosamente en tareas de remediación (con biomasa viva o muerta). Así lo demuestran numerosos trabajos que evalúan el uso de distintas macroalgas en la remoción de uranio, cobre, cadmio, arsénico, zinc e incluso compuestos orgánicos (Cheney et al. 2014; Anacleto et al. 2017; Bagda et al. 2017; Deniz y Karabulut 2017; Flores-Chaparro et al. 2017, 2018; Vieira et al. 2017; Poo et al. 2018). A nivel local, muchos trabajos evidencian las potencialidades de las macroalgas argentinas en la remoción de distintos contaminantes del agua. Basso et al. (2002) examinaron la capacidad para remover $\mathrm{Cd}$ de aguas contaminadas de las macroalgas Corallina officinalis L, P. columbina y Codium fragile de la costa atlántica del sur argentino. Los resultados mostraron que Coralina y Porphira presentaron una alta efectividad ( $>$ 90\%), superior a la determinada para Codium y para el carbón activado comercial. Además, las 
pruebas experimentales realizadas por Areco y dos Santos Afonso (2010) y Areco et al. (2012), sugieren que la biomasa de Gymnogongrus torulosus y U. lactuca (ambas colectadas en Mar del Plata) es un bioadsorbente eficaz para la eliminación de los iones $\mathrm{Zn}$ (II), $\mathrm{Cu}(\mathrm{II}), \mathrm{Pb}(\mathrm{II})$ y $\mathrm{Cd}(\mathrm{II})$, por lo que podría ser una tecnología alternativa y de bajo costo para el tratamiento de efluentes industriales. Plaza-Cazón et al. (2011, 2012a, 2012b, 2013) evaluaron la capacidad de dos especies de algas pardas, $U$. pinnatifida y $M$. pyrifera extraídas de Bahía de Camarones y Golfo Nuevo (Patagonia argentina), como biosorbente para la remoción de $\mathrm{Hg}(\mathrm{II}), \mathrm{Cr}(\mathrm{III}), \mathrm{Zn}(\mathrm{II})$ y $\mathrm{Cd}(\mathrm{II})$ de soluciones acuosas. Los resultados mostraron que las algas estudiadas presentan diferencias significativas en sus propiedades de biosorción de $\mathrm{Hg}(\mathrm{II})$, pero no de $\mathrm{Cr}(\mathrm{III})$, y que son un adsorbente adecuado para la remoción de Cd(II) y Zn(II). Por otro lado, Carnevale et al. (2016) utilizaron Petalonia fascia recolectada de las costas de Puerto Madryn (Chubut, Argentina) para el tratamiento de aguas subterráneas y efluentes contaminados con molibdeno. Sus resultados demuestran que especialmente las algas pardas tienen un gran potencial como biosorbente debido a su alta eficiencia en la captación de iones metálicos y su bajo costo. Por último, hay trabajos que combinan las macroalgas con otros materiales, por ejemplo, nanopartículas de hierro para la remoción de colorantes con excelentes resultados (García et al. 2018).

\section{Aplicaciones en la industria farmacéutica y medicina}

Las macroalgas son fuente de una gran variedad de moléculas y compuestos químicos denominados nutracéuticos, muy valorados por la industria farmacéutica y cosmética. Nutracéutico es un término derivado de las palabras "nutrición" y "farmacéutico", y podría definirse como "cualquier sustancia que sea un alimento o parte de un alimento y proporcione beneficios médicos o a la salud, incluyendo la prevención y el tratamiento de enfermedades" (DeFelice 2002). Muchos trabajos han demostrado las excelentes propiedades antioxidantes, antitumorales, antibacteriales, anticancerígenas, entre otras, que poseen los extractos de diferentes algas (Hardouin et al. 2016; Pérez et al. 2016; Ammar et al. 2017; Mathew et al. 2017). Numerosos estudios evidencian las propiedades nutracéuticas de las macroalgas argentinas. Debido a los altos niveles de proteínas de las algas, son fuente de péptidos bioactivos con interesantes funciones fisiológicas. Cian et al (2018) encontraron que los péptidos presentes en Ulva sp. ejercen efectos antiinflamatorios en células inmunes. Por otro lado, las algas rojas son ricas en polisacáridos sulfatados con potenciales efectos terapéuticos: actividad antioxidante, anticoagulante, anticancerígena, antibacterial y antiviral. En concordancia con otras investigaciones (Sanjeewa et al. 2018; Alencar et al. 2019), en la Argentina se ha encontrado que extractos obtenidos a partir del alga Iridaea undulosa tienen efectos antitumorales (Calvo et al. 2019), y que extractos del alga roja Gracilariopsis hommersandii poseen propiedades biológicas interesantes como potenciales agentes anticoagulantes, antioxidantes, antitumorales y antivirales (Rodríguez-Sánchez et al. 2019). También el alga marrón Scytosiphon lomentaria produce fucoidanos con actividad antiviral alta y selectiva (Ponce et al. 2019). Varios grupos argentinos abordan el estudio químico de los fucoidanos y galactanos producidos por las algas (Estevez et al. 2004; Perez-Recalde et al. 2016).

Por otra parte, existe un creciente interés en el desarrollo de biomateriales provenientes de fuentes renovables para aplicaciones en medicina regenerativa. Un material adecuado para la ingeniería de tejidos debe cumplir algunos requisitos en materia de biocompatibilidad, degradabilidad y toxicidad. Además, el material debe ser accesible, estar disponible y tener un costo de fabricación relativamente bajo. Torres et al. (2019) encontraron que el alginato extraído de U. pinna- 
tifida es un material adecuado para estos fines, $\mathrm{y}$ que su purificación mejora la biocompatibilidad y la regeneración ósea, al mismo tiempo que disminuye su toxicidad.

\section{Aplicaciones en la industria energética}

Las algas son una fuente prometedora para la producción de energía renovable, ya que pueden fijar las emisiones de gases de efecto invernadero, y principalmente porque no compiten con la producción de alimentos (Chen et al. 2015). En su trabajo, Aitken et al. (2014) resaltan el reciente interés sobre la producción de biocombustible a partir de biomasa algal y sus potenciales beneficios ambientales. Los autores encontraron que el cultivo y procesamiento de algas marinas para la obtención de bioetanol y electricidad a partir del biogás producido por Gracilaria chilensis y $M$. pyrifera podría ser rentable. En el mismo sentido se encuentran otros trabajos más recientes (Fan et al. 2015; Ghadiryanfar et al. 2016).

En la búsqueda de energías limpias y económicas, surge otra aplicación para las algas marinas: su uso en la fabricación de células solares para la producción de energía fotovoltaica basada en la obtención de energía eléctrica a partir de energía lumínica. Para ello, diferentes materiales denominados semiconductores se cubren con colorantes que incrementan la captación de luz. Los colorantes a base de clorofila obtenida a partir de algas marinas representan una alternativa económica y de fácil extracción y almacenamiento (Calogero et al. 2014). Se reportan escasas investigaciones argentinas en este eje temático. Por su parte, Garriga et al. (2017) hacen un aporte sobre el potencial de las algas como fuente de biocombustibles líquidos, mediante la conversión de los carbohidratos de la biomasa algal en bioetanol, proponiendo un método analítico para la determinación de azúcares. Otro trabajo, evalúa la variación estacional de la composición química de U. pinnatifida (Puerto Madryn) en relación con su uso para la producción de bioetanol. Sus resultados muestran que las algas recogidas en verano son un recurso potencial con un alto contenido de azúcares totales, bajo contenido graso y bajo valor proteico, adecuado para la producción de bioetanol (Mendes et al. 2019). Además, mencionan que en particular el uso industrial de $U$. pinnatifida para obtener el bioetanol podría contribuir sustancialmente a la reducción del impacto ambiental y económico que genera su presencia en las costas patagónicas. Los trabajos evidencian una aplicación viable que puede ser interesante desarrollar.

\section{Aplicaciones en la agricultura}

En la agricultura, las macroalgas marinas muestran propiedades como fertilizantes naturales y bioestimulantes. Los extractos de algas son utilizados como reguladores de crecimiento, contienen altos niveles de hormonas vegetales, en particular citoquinonas, además de polisacáridos, aminoácidos y macro y micronutrientes necesarios para el crecimiento y desarrollo de las plantas (Craigie 2011). Varios trabajos evidencian los efectos positivos en el crecimiento y la composición de ciertos cultivos debido a la aplicación de extractos preparados a partir de algas marinas (Ciepiela et al. 2016). También hay evidencias de que el suministro de estos bioestimulantes influye positivamente en el estado general de la planta aumentando su resistencia a enfermedades y plagas (De Corato et al. 2017).

En el caso particular del uso de algas para la producción de compost, resulta interesante destacar que puede utilizarse material algal descartado de otros usos e incluso las algas marinas producto del arribazón (Eyras y Sar 2003; Piriz et al. 2003). Un artículo realizado sobre arribazones en la Provincia de Buenos Aires proporciona valiosa información acerca de la composición de las comunidades submareales y permite evaluar su potencialidad de utilización (Becherucci y Benavides 2016). La ventaja frente a otros compost es que la biomasa algal posee una baja relación $\mathrm{C}: \mathrm{N}$, lo que 
aumenta la calidad del fertilizante (Eyras y Rostagno 1995). Además, los autores comprobaron que el agregado de algas mejoró notablemente las propiedades físicas de los suelos (menor densidad aparente y mayor tasa de infiltración). Posteriormente, Eyras et al. (2008) comprobaron el aumento del rendimiento y la resistencia a las enfermedades de las plantas de tomate debido a la adición de compost de algas marinas. Por su parte, Cole et al. (2016) señalan que el compost preparado con algas es una alternativa ecológica interesante a los fertilizantes sintéticos. Sus estudios mostraron que los cultivos de cañas de azúcar fertilizados con compost de algas marinas generaban 4 veces más biomasa que aquellos obtenidos utilizando un compost comercial sin algas.

\section{CONCLUSIONES}

La presente revisión demuestra que las macroalgas marinas presentan aplicaciones en numerosos campos. En cuanto a los beneficios de su consumo para la salud humana, los trabajos coinciden en las ventajas que representa incluir algas marinas en la dieta. Sin embargo, se requiere profundizar los estudios sobre composición química, en especial aquellos focalizados en el contenido de elementos potencialmente tóxicos, así como también interpelarse sobre el contenido máximo permitido de los distintos elementos en algas comestibles. Respecto a los resultados vinculados a las aplicaciones ambientales, se coincide en que el uso de la fitorremediación con macroalgas puede ser una estrategia de mitigación útil y efectiva para disminuir los niveles de contaminantes en el agua de mar, efluentes y aguas residuales. Además, apoyan la idea de que las algas son eficaces indicadores de la salud de los ecosistemas marinos. Cabe destacar, que las investigaciones en este campo están orientadas fundamentalmente a la presencia de elementos potencialmente tóxicos en el ambiente; sin embargo, la ocurrencia de com- puestos orgánicos en los cuerpos de agua es un tema de creciente interés. Identificamos entonces un aspecto que aún no ha recibido adecuada atención. Por otra parte, la mayoría de los estudios en la Argentina proponen experimentos de laboratorio para el tratamiento de soluciones acuosas, y muy pocos estudios abordan investigaciones a escalas piloto y/o con aguas reales, lo que implica un nivel de complejidad mayor pero fundamental para su aplicación viable. En relación con su aplicación en el campo de la salud, la literatura presentada aquí demuestra claramente que las algas marinas son fuente de compuestos bioactivos con múltiples beneficios para la salud, y que esta es un área ampliamente estudiada. Además, se evidencia el uso promisorio de las algas en la producción de biocombustible, aunque es un campo que aún debe explorarse más. Respecto a su uso en agricultura, queda demostrado que la aplicación de extractos algales influye positivamente en el estado general de la planta, aumenta su resistencia a enfermedades y plagas y mejora el rendimiento de cultivos alimenticios, así como también es una alternativa interesante como fertilizante. En este sentido, grandes cantidades de algas podrían ser reutilizadas en lugar de ser desechadas. La ampliación de sus usos aumentaría la demanda de algas marinas $\mathrm{y}$, en consecuencia, las economías de las poblaciones costeras que las producen. Por último, las algas argentinas tienen un gran potencial en usos alimenticios, industria farmacéutica y aplicaciones ambientales como se concluye de los trabajos reportados.

\section{AGRADECIMIENTOS}

Este trabajo se realizó en el marco del proyecto de la Agencia Nacional de Promoción Científica y Tecnológica PICT 2016-2718. Los autores agradecen al Instituto de Investigación e Ingeniería Ambiental de la Universidad Nacional de San Martín. 


\section{REFERENCIAS}

Afonso C, Cardoso C, Ripol A, Varela J, Quental-Ferreira H, Pousao-Ferreira P, Ventura MS, Delgado IM, Coelho I, CasTANHEIRA I, BANDARRA NM. 2018. Composition and bioaccessibility of elements in Green seaweeds from fish pond aquaculture. Food Res Int. 105: 271-277.

Aitken D, Balboa C, Godoy-Faundez A, Turrion-Gomez JL, Antizar-Ladislao B. 2014. Life cycle assessment of macroalgae cultivation and processing for biofuel production. $\mathrm{J}$ Clean Prod. 75: 45-56.

Alencar POC, Lima GC, Barros FCN, Costa LEC, Ribeiro CVPE, Sousa WM, Sombra VG, Abreu CMWS, Abreu ES, Pontes EOB, et al. 2019. A novel antioxidant sulfated polysaccharide from the algae Gracilaria caudata: In vitro and in vivo activities. Food Hydrocoll. 90: 28-34.

Areco MM, dos SAntos Afonso M. 2010. Copper, zinc, cadmium and lead biosorption by Gymnogongrus torulosus. Thermodynamics and kinetics studies. Colloids Surf. B: Biointerfaces. 81: 620-628.

Areco MM, Hanela S, Duran J, dos Santos Afonso M. 2012. Biosorption of $\mathrm{Cu}(\mathrm{II})$, $\mathrm{Zn}(\mathrm{II}), \mathrm{Cd}(\mathrm{II})$ and $\mathrm{Pb}(\mathrm{II})$ by dead biomasses of green alga Ulva lactuca and the development of a sustainable matrix for adsorption implementation. J Hazard Mater. 213-214: 123-132.

Alves RN, Maulvault AL, Barbosa VL, FerNANDEZ-TeJedor M, Tediosi A, KotTerman M, van del Heuvel FHM, Robbens J, FerNANDES JO, RASMUSSEN RR, et al. 2018. Oral bioaccessibility of toxic and essential elements in raw and cooked commercial seafood species available in European markets. Food Chem. 367: 15-27.

Amin MHA, Xiong C, Glabonjat RA, Francesconi KA, Oguri T, Yoshinaga J.
2018. Estimation of daily intake of arsenolipids in Japan based on a market basket survey. Food Chem Toxicol. 118: 245-251.

Ammar N, Jabnoun-Khiareddine H, MejdoubTrabelsi B, Nefzi A, Mahjoub MA, DaAmiREMADI M. 2017. Pythium leak control in potato using aqueous and organic extracts from the brown alga Sargassum vulgare (C. Agardh, 1820). Postharvest Biol Technol. 130: 81-93.

Anacleto P, van den Heuvel fHM, Oliveira C, Rasmussen RR, Fernandes JO, Sloth JJ, Barbosa V, Alves RN, Marques A, Cunha SC. 2017. Exploration of the phycoremediation potential of Laminaria digitata towards diflubenzuron, lindane, copper and cadmium in a multitrophic pilot-scale experiment. Food Chem Toxicol. 104: 95-108.

Astorga-EsPaÑa MS, RodríGUEZ-GALdÓN B, RodríGuEZ-RodríGuEz EM, DíAz-RoMERo C. 2016. Aminoacid content in seaweeds from the Magellan Straits (Chile). J Food Compos Anal. 53: 77-84.

Bagda E, Tuzen M, Sari A. 2017. Equilibrium, thermodynamic and kinetic investigations for biosorption of uranium with green algae (Cladophora hutchinsiae). J Environ Radioactiv. 175-176: 7-14.

Balboa EM, Gallego-Fábrega C, Moure A, Dominguez H. 2015. Study of the seasonal variation on proximate composition of ovendried Sargassum muticum biomass collected in Vigo Ria, Spain. J Appl Phycol. 28 (3): 1943-1953.

BARONI L, Goggi S, BAtTAglino R, BerVEglieri M, FASAn I, FilipPin D, GRIFFITH P, Rizzo G, Tomasini C, Tosatti MA, BAtTino MA. 2019. Vegan nutrition for mothers and children: practical tools for healthcare providers. Nutrients. 11 (5): 1-16.

Basso MC, Cerrella EG, Cukierman. 2002. Empleo de algas marinas para la biosorción de metales pesados de aguas contaminadas. Avances en Energías Renovables y Medio 
Ambiente. 6 (1): 60-74.

Becherucci ME, Benavides H. 2016. Composición florística de arribazones de algas marinas en playas del sudeste de la provincia de Buenos Aires, Argentina. Iheringia Ser Bot. 71 (1): $1-5$.

Bonanno G, Orlando-Bonaca M. 2018. Chemical elements in Mediterranean macroalgae. A review. Ecotox Environ Safe. 148: 44-71.

Boraso de Zaixso, AL, Ciancia M, Cerezo AS, Piriz ML, Casas GN, Eyras MC. 2015. Capítulo 1: Utilización de las macroalgas marinas de la costa argentina y sus hidrocoloides. En: Zaixso HE, Boraso AL, editores. La Zona Costera Patagónica Argentina. Vol. I. Recursos Biológicos Bentónicos. Editorial Universitaria de la Patagonia. Comodoro Rivadavia. Versión digital: 3-60.

Calogero G, Citro I, Di Marco G, Minicante SA, Morabito M, Genovese G. 2014. Brown seaweed pigment as a dye source for photoelectrochemical solar cells. Spectrochim Acta A. 117: 702-706.

Calvo GH, Cosenza VA, Sáenz DA, Navarro DA, Stortz CA, Céspedes MA, Mamone LA, Casas AG, Di Venosa GM. 2019. Disaccharides obtained from carrageenan as potential antitumor agents. Sci Rep. 9 (6654): 1-13.

Camurati JR, Salomone VN. 2019. Arsenic in edible macroalgae: an integrated approach. Journal of Toxicology and Environmental Health, Part B: Critical Reviews. doi: 10.1080/10937404.2019.1672364

Carnevale B, Blanes P, Sala lF, Bellú SE. 2017. Removal of molybdate anions from contaminated waters by brown algae biomass in batch and continuous processes. J Chem Technol Biotechnol. 92: 1298-1305.

CAsas G, Piriz ML. 1996. Survey of Undaria pinnatifida (Laminariales, Phaeophyta) in Golfo Nuevo, Argentina. Hydrobiologia. 326/327: 213-215.

Casas GN, Scrosati R, Piriz ML. 2004. The invasive kelp Undaria pinnatifida (Phaeo- phyceae, Laminariales) reduces native seaweed diversity in Nuevo Gulf (Patagonia, Argentina). Biol Inv. 6: 411-416.

Chen H, Zhou D, Luo G, Zhang S, Chan J. 2015. Macroalgae for biofuels production: progress and perspectives. Renew Sust Energ Rev. 47: 427-437.

Cheney D, Rajic L, Sly E, Merci D, Sheahan A. 2014. Uptake of PBCs contained in marine sediments by the green macroalga Ulva rigida. Mar Pollut Bull. 88: 207-214.

Cian RE, Bacchetta C, Rossi A, Cazenave J, Drago SR. 2019. Red seaweed Pyropia columbina as antioxidant supplement in feed for cultured juvenile Pacú (Piaractus mesopotamicus). J Appl Phycol. 31: 1455-1465.

Cian RE, Hernández-Chirlaque C, GámezBelmonte R, Drago SR, Sánchez de MediNA F, Martínez-Augustín O. 2018. Green alga Ulva spp. hydrolysates and their peptide fractions regulate cytokine production in splenic macrophages and lymphocytes the TLR4-NFkB/MAPK pathways. Mar Drugs. 16: 235-249.

Circuncisào AR, CATARINo MD, CARdoso SM, SILVA AMS. 2018. Minerals from macroalgae origin health benefits and risks for consumers. Mar Drugs. 16: 400-430.

Ciepiela GA, Godlewska A, Jankowska J. 2016. The effect of seaweed Ecklonia maxima extract and mineral nitrogen on fodder grass chemical composition. Environ Sci Pollut R. 23 (3): 2301-2307.

Cofrades S, Benedí J, Garcimartin A, SÁNCHEZ-MuÑiz FJ, JiMENEZ-COLMENERO F. 2017. A Comprehensive approach to formulation of seaweed-enriched meat products: from technological development to assessment of healthy properties. Food Res Int. 99: 10841094.

Cofrades S, López-López I, Bravo L, RuizCapillas C, Bastida S, Larrea MT, JiménezColmenero F. 2010. Nutritional and antioxidant properties of different Brown and red 
Spanish edible seaweed. Food Sci Technol Int. 16 (5): 361-370.

Cole AJ, Roberts DA, Garside A, De Nys R, PAUL NA. 2016. Seaweed compost for agricultural crop production. J Appl Phycol. 28: 629642.

Craigie JS. 2011. Seaweed extract stimuli in plant science and agriculture. J Appl Phycol. 23 (3): 371-393.

De Corato U, Salimbeni R, De Pretis A, AveLla N, Patruno G. 2017. Antifungal activity of crude extracts from brown and red seaweeds by supercritical carbon dioxide technique against fruit postharvest fungal diseases. Postharvest Biol Technol. 131: 16-30.

DeFelice S. 2002. FIM, Rationale and Proposed Guidelines for the Nutraceutical Research and Education Act NREA, Foundation for Innovation in Medicine. http:/www.fimdefelice.org/ archives/arc.researchact.html.

Dellatorre FG, Amoroso R, Saravia J, OrenSANZ JM. 2014. Rapid expansion and potential range of the invasive kelp Undaria pinnatifida in the Southwest Atlantic. Aquat Invasions. 9 (4): 467-478.

Deniz F, Karabulut A. 2017. Biosorption of heavy metals ions by chemically modified biomass of coastal seaweed community: Studies on phycoremediation system modeling and design. Ecol Eng. 106: 101-108.

Diana JS, Egna HS, Chopin T, Peterson MS, Cao L, Pomeroy R, Verdegem M, Slack WT, Bondad-Reantaso MG, Cabello F. 2013. Responsible Aquaculture in 2050: Valuing local conditions and human innovations will be key to success. BioScience. 63 (4): 255-262.

Diaz AC, Espino ML, Arzoz NS, Velurtas SM, Ponce NMA, Stortz CA, Fenucci JL. 2017. Free radical scavenging activity of extracts from seaweeds Macrocystis pyrifera and Undaria pinnatifida: applications as functional food in the diet of prawn Artemesia longinaris. Lat Am J Aquat Res. 45 (1): 104-112.
Estevez JM, Ciancia M, Cerezo AS. 2004. The system of galactans of the red seaweed, Kappaphycus alvarezii, with emphasis on its minor constituents. Carbohydr Res. 339: 2575-2592.

Eyras MC, Defosse GE, Dellatorre F. 2008. Seaweed compost as an amendment for horticultural soils in Patagonia, Argentina. Compost Sci Util. 16 (2): 119-124.

Eyras MC, Rostagno CM. 1995. Bioconversión de algas marinas de arribazón: experiencias en Puerto Madryn, Chubut (Argentina). Naturalia Patagónica, ciencias Biológicas. 3: 25-39.

Eyras MC, SAR EA. 2003. Arribazones estivales en Puerto Madryn, Argentina, como materiales para la obtención de compost. Bol Soc Argent Bot. 38 (1-2): 105-111.

FAN X, GUO R, YUAN X, QIU Y, YANG Z, WANG F, Sun M, ZhaO X. 2015. Biogas production from Macrocystis pyrifera biomass in seawater system. Bioresource Technol. 197: 339347.

[FAO] Organización de las Naciones Unidas PARA LA AlimENTACIÓN Y LA AgRicultura. 2018. El estado mundial de la pesca y la acuicultura 2018. Cumplir los objetivos de desarrollo sostenible. Roma. Licencia: CC BYNC-SA 3.0 IGO.

Farías DR, Hurd CL, Eriksen RS, Macleod CK. 2018. Macrophytes as bioindicators of heavy metal pollution in estuarine and coastal environments. Mar Pollut Bull. 128: 175-184.

Farías S, Pérez Arisnabarreta S, Vodopidez C, Smichowski P. 2002. Levels of essential and potentially toxic trace metals in Antarctic macro algae. Spectrochim Acta B. 57: 21332140.

Farías S, Smichowski P, Vélez D, Montoro R, Curtosi A, Vodopívez C. 2007. Total and inorganic arsenic in Antartic macroalgae. Chemosphere. 69: 1017-1024.

Flores-Chaparro CE, Chazaro Ruiz LF, Alfaro-De la Torre MA, Huerta-Diaz A, RanGEL-MENDEZ JR. 2017. Biosorption removal 
of benzene and toluene by three dried macroalgae at different ionic strength and temperatures: algae biochemical composition and kinetics. J Environ Manag. 193: 126-135.

Flores-Chaparro CE, Rodriguez-HernandeZ MC, Chazaro-Ruiz LF, Alfaro-De la Torre MA, Huerta-Diaz MA, Rengel-Mendez JR. 2018. Chitosan-macroalgae biocomposites as potential adsorbents of water-soluble hydrocarbons: organic matter and ionic strength effects. J Clean Prod. 197: 633-642.

Gallardo AA, Risso S, Fajardo MA, Belchior SE. 2004. Caracterización de poblaciones microbianas presentes en la macroalga comestible Mosotroma undulatum, Wittrock. Archivos Latinoamericanos de Nutrición. 34 (3): 117.

García FE, Plaza-Cazón J, Montesinos VN, DONATi ER, LiTTER MI. 2018. Combined strategy for removal of Reactive Black 5 by biomass sorption on Macrocystis pyrifera and zerovalent iron nanoparticles. J Environ Manage. 207: 70-79.

García-SARTAL C, BARCIEla-Alonso MC, Moreda-Piñeiro A, Bermejo-Barrera P. 2013. Study of cooking on the bioavailability of $\mathrm{As}, \mathrm{Co}, \mathrm{Cr}, \mathrm{Cu}, \mathrm{Fe}, \mathrm{Ni}$, Se and $\mathrm{Zn}$ from edible seaweed. Microchem J. 108: 92-99.

Garriga M, Almaraz M, Marchiaro A. 2017. Determination of reducing sugars in extracts of Undaria pinnatifida (harvey) algae by UVvisible spectrophotometry (DNS method). Actas Ing. 3: 173-179.

GHADIRYANFAR M, ROSENTRATER KA, KAYHANI A, ОміD M. 2016. A review of macroalgae production, with potential applications in biofuels and bioenergy. Renew Sus Energ Rev. 54: 473-481.

Gil MN, Torres AI, Commendatore MG, Marinho C, Arias A, Giarratamo E, Casas GN. 2015. Nutritive and xenobiotic compounds in the alien algae Undaria pinnatifida from argentine Patagonia. Arch Environ Contam Toxicol. 68: 553-565.
Gil MN, Torres AI, Esteves JL. 2005. Uptake of sewage derived nitrogen by Ulva rigida (Chlorophyceae) in Bahía Nueva (Golfo Nuevo, Patagonia, Argentina). Hydrobiologia. 532: 39-43.

Hardouin K, Bedoux G, Burlot AS, DonnayMoreno C, Bergé JP, Nyvall-Collén P, Bourgougnon N. 2016. Enzyme-assisted extraction (EAE) for the production of antiviral and antioxidant extracts from the green seaweed Ulva armoricana (Ulvales, Ulvophyceae). Algal Res. 16: 233-239.

JAMES K. 2017. A review of the impacts from invasion by the introduced kelp Undaria pinnatifida. Waikato Regional Council Technical Report 2016/40: 32 p.

KaRTHiCK P, Siva SANKAR R, KaViarasan T, MOHANRAJU. 2012. Ecological implications of trace metals in seaweeds: Bio-indication potential for metal contamination in Wandoor, South Andaman Island. Egyptian J Aquat Res. 38: 227-231.

Ma Z, Lin L, Wu M, Yu M, Shang T, Zhang T, ZHAO M. 2018. Total and inorganic arsenic contents in seaweeds: absorption, accumulation, transformation and toxicity. Aquaculture. 497: 49-55.

Makkar HPS, Tran G, Heuzé V, GigerREVERDIN S, LESSIRE M, LEBAS F, DP ANKERS. 2016. Seaweeds for livestock diets: a review. Anim Feed Sci Tech. 212: 1-17.

Martin JP, Cuevas JM. 2006. First record of Undaria pinnatifida (Laminariales, Phaeophyta) in Southern Patagonia, Argentina. Biol Invasions. 8: 1399-1402.

Marzocchi M, Badocco D, Plovan A, Pastore P. 2016. Metals in Undaria pinnatifida (Harvey) Suringar and Sargassum muticum (yendo) Fensholt edible seaweeds growing around Venice (Italy). J Appl Phycol. 28: 2605-2613.

Mathew L, Burney M, Gaikwad A, Nyshadha P, Nugent EK, Gonzalez A, Smith JA. 2017. Preclinical evaluation of safety of fucoindan 
extracts from Undaria pinnatifida and Fucus vesiculosus for use in cancer treatment. Integr Cancer Ther. 16 (4): 572-584.

Mendes Rosa M, Maldonado FL, Marchiaro AB. 2019. Seasonal variation in the chemical composition of seaweed Undaria pinnatifida. Academia J Sci Res. 7 (2): 134-138.

Meretta PE, Matula CV, Casas G. 2012. Occurrence of the alien kelp Undaria pinnatifida (Laminariales, Phaeophyceae) in Mar del Plata, Argentina. Bio Inv Rec. 1 (1): 59-63.

Miedico O, Pompa C, Tancredi C, Cera A, Pellegrino E, Tarallo M, Chiaravalle AE. 2017. Characterisation and chemometric evaluation of 21 trace elements in three edible seaweed species imported from south-east Asia. J Food Comp Anal. 64: 188-197.

MuÑOZ J. 2015. Informe final: Incorporación de la industria alimentaria de consume humano directo como Fuente de agregación de valor para las macroalgas nacionales. Universidad Arturo Prat. Instituto de Ciencia y Tecnología (ICYT), Puerto Montt, Chile. 411 p.

Muse JO, Carducci CN, Stripeikis JD, Tudino MB, Fernámdez FM. 2006. A link between lead and cadmium kinetic speciation in seawater and accumulation by green alga Ulva lactuca. Environ Pollut. 141: 126-130.

Muse JO, Tudino MB, D'Huicque L, Troccoli OE, CARDUCCI CN. 1989. Atomic Absorption Spectrometric determination of inorganic and organic arsenic in some marine benthic algae of the Southern Atlantic Coast. Environ Pollut. 58: 303-312.

Paz S, Rubio C, Frías i, Gutiérrez AJ, Gonzáles-Weller D, Martín V, Revert C, HARDisSON A. 2019. Toxic metals (Al, Cd, Pb and $\mathrm{Hg}$ ) in the most consumed edible seaweeds in Europe. Chemosphere. 218: 879884.

Paz S, Rubio C, Gutiérrez AJ, GonzálezWeller D, Revert C, Hardisson A. 2018. Metal concentrations in wild-harvested phaeophyta seaweed from the Atlantic Ocean
(Canary Island, Spain). J Food Protect. 81 (7): 1165-1170.

Pérez AA, Farías SS, Strobl AM, Pérez LB, López CM, Piñeiro A, Roses O, Fajardo MA. 2007. Levels of essential and toxic elements in Porphyra columbina and Ulva sp. from San Jorge Gulf, Patagonia Argentina. Sci Total Environ. 376: 51-59.

Pérez AA, Pérez lB, Strobl AM, Camarda S, FARIAS SS, López CM, Fajardo MA. 2010. Variación estacional de arsénico total en algas comestibles recolectadas en el Golfo San Jorge (Chubut, Argentina). Rev Latinoam Biotecnol Amb Algal. 1 (1): 16-30.

Pérez MJ, Falqué E, Dominguez H. 2016. Antimicrobial action of compounds from marine seaweed. Mar Drugs. 14: 52-90.

Pérez Recalde M, Canelón DJ, Campagnone RS, Matulewicz MC, Cerezo AS, Ciancia M. 2016. Carrageenan and agaran structures from the red seaweed Gymnogongrus tenuis. Carbohyd Polym. 136: 1370-1378.

Piriz ML, Eyras MC, Rostagno CM. 2003. Changes in biomass and botanical composition of beach-cast seaweeds in a disturbed coastal area from Argentine Patagonia. J Appl Phycol. 15 (1): 67-74.

Plaza-Cazón J, Benitez L, Donati E, Viera M. 2012a. Biosorption of chromium(III) by two Brown algae Macrocysits pyrifera and Undaria pinnatifida: Equilibrium and kinetic study. Eng Life Sci. 12 (1): 95-103.

Plaza-Cazón J, Bernardelli C, Viera M, Donati E, Guibal E. 2012b. Zinc and cadmium biosorption by untreated and calciumtreated Macrocystis pyrifera in a batch system. Bioresourse Technol. 116: 195-203.

Plaza-Cazón J, Viera M, Donati E, Guibal E. 2011. Biosorption od mercury by Macrocystis pyrifera and Undaria pinnatifida: influence of zinc, cadmium and nickel. J Environ Sci. 23 (11): 1778-1786.

Plaza-Cazón J, Viera M, Donati E, Guibal E. 2013. Zinc and cadmium removal by biosorp- 
tion on Undaria pinnatifida in batch and continuous processes. J Environ Manage. 129: 423-434.

Ponce nMa, Flores mL, Pujol CA, Becerra MB, Navarro DA, Córdoba O, Damonte EN, Stortz CA. 2019. Fucoidans from the phaeophyta Scytosipho lomentaria: Chemical analysis and antiviral activity of the galactofucan component. Carbohyd Res. 478: 18-24.

Poo K, Son E, Chang J, Ren X, Choi Y, Chae K. 2018. Biochars derived from wasted marine macro-algae (Saccharina japonica and Sargassum fusiforme) and their potential for heavy metal removal in aqueous solution. J Environ Manage. 206: 364-372.

Rebours C, Marinho-Soriano E, ZertucheGonzalez JA, Hayashi L, VÁsquez JA, KRADOlfer P, Soriano G, Ugarte R, Abreu MH. 2014. Seaweeds: an opportunity for wealth and sustainable livehood for coastal communities. J Appl Phycol. 26: 1939-1951.

Rodrigues D, Freitas AC, Pereira L, RochaSAntos TAP, VASCONCElos MW, Roriz M, RodríGUEZ-AlCAlÁ LM, GoMES AMP, DuARTE AC. 2015. Chemical composition of red, brown and green macroalgae from Buarcos bay in Central West Coast of Portugal. Food Chem. 183: 197-207.

RodRÍGUEZ-SÁNCHEZ RA, CANELÓN DJ, COSENZA VA, Fissore EN, Gerschenson LN, MATUleWICZ MC, CiAnCiA M. 2019. Gracilariopsis hommersandii, a red seaweed, source of agar and sulfated polysaccharides with unusual structures. Carbohyd Polym. 213: 138-146.

Rubio C, Napoleone G, Luis-González G, Gutiérrez AJ, GonzÁlez-Weller D, HarDisson A, REVERT C. 2017. Metals in edible seaweed. Chemosphere. 173: 572-579.

Salomone VN, Riera M. 2019. Proximal composition of Undaria pinnatifida from San Jorge Gulf (Patagonia, Argentina). Biol Trace Elem Res. doi: 10.1007/s12011-019-01905-1

Salomone VN, Riera M, Cerchietti L, Custo G, Muniain C. 2017. Seasonal determination of trace and ultra-trace content Macrocystis pyrifera from San Jorge Gulf (Patagonia) by Total Reflection X-ray Fluorescence. Spectrochim Acta B. 131: 74-78.

Sanjeewa KKA, Kang N, Ahn G, Jee Y, Kim Y, JEON Y. 2018. Bioactive potentials of sulfated polysaccharides isolated from brown seaweed Sargassum spp in related to human health applications: a review. Food Hydrocoll. 81: 200-208.

SCHMid M, KRAFT LGK, VAN DEL LoOS LM, Kraft GT, Virtue P, Nichols PD, Hurd CL. 2018. Southern Australian seaweed: a promising resource for omega-3 fatty acids. Food Chem. 265: 70-77.

SKIRZYPCZYK VM, HERMON KM, NORAMBUENA F, Turchini GM, Keast R, Bellgrove A. 2019. Is Australian seaweed worth eating? Nutritional and sensorial properties of wild-harvested Australian versus commercially available seaweeds. J Appl Phycol. 31 (1): 709724.

Taboada MC, Millán R, Miguez MI. 2013. Nutritional value of the marine algae wakame (Undaria pinnatifida) and nori (Porphyra purpurea) as food supplements. J Appl Phycol. 25: 1271-1276.

Taylor V, Goodale B, RaAb A, Schwerdtle T, Reimer K, Conklin S, Karagas MR, FRANCESCONI KA. 2017. Human exposure to organic arsenic species from seafood. Sci Total Environ. 580: 266-282.

TAYLOR VF, JACKSON BP. 2016. Concentrations and speciation of arsenic in New England seaweed species harvested for food and agriculture. Chemosphere. 163: 6-13.

Torres ML, Fernandez JM, Dellatorre FG, Cortizo AM, Oberti TG. 2019. Purification of alginate improves its biocompatibility and eliminates cytotoxicity in matrix for bone tissue engineering. Algal Res. 40. doi:10.1016/j. algal.2019.101499

Torres AI, Gil MN, Esteves JL. 2004. Nutrient uptake rates by the alien alga Undaria pinnat- 
ifida (Phaeophyta) (Nuevo Gulf, Patagonia, Argentina) when exposed to diluted sewage effluent. Hydrobiologia. 520: 1-6.

VieIRA BRC, Pintor AMA, Bonaventura RAR, Botelho CMS, Santos SCR. 2017. Arsenic removal from water using iron-coated seaweed. J Environ Manage. 192: 224-233.

WaLls AM, KenNedy R, EdWARds MD, JOHNSON MP. 2017. Impact of kelp cultivation on the Ecological Status of benthic habitats and
Zostera marina seagrass biomass. Mar Pollut Bull. 123: 19-27.

Zhou Q, Zhang J, Pu J, Shi J, Jiang G. 2008. Biomonitoring: an appealing tool for assessment of metal pollution in the aquatic ecosystem. Anal Chim Acta. 606: 135-150.

Recibido: 04-10-2019

Aceptado: 26-11-2019 
